\title{
ESTRATEGIAS INNOVADORAS PARA FAVORECER EL INGRESO Y LA PERMANENCIA EN CARRERAS STEM
}

Dapozo, Gladys N.; Greiner, Cristina L.; Petris, Raquel H.

\section{RESUMEN}

Los profesionales formados en disciplinas STEM (Ciencia, Tecnología, Ingeniería y Matemáticas) utilizan sus conocimientos para entender cómo funciona el mundo y resolver problemas, utilizandocomputadoras y otras herramientas tecnológicas. La problemática que se plantea a nivel mundial es una marcada disminución de estudiantes en estas carreras, y escasez de recursos humanos calificados, cuando paradójicamente son altamente demandados. En este trabajo se presenta una reseña de los esfuerzos institucionales que se realizan para apoyar a los ingresantes de las carreras STEM que se ofrecen en esta unidad académica $\mathrm{y}$ se describe una experiencia innovadora de incorporación de herramientas lúdicas como modalidad de aprestamiento para los ingresantes a la carrera Licenciatura en Sistemas de Información de la Universidad Nacional del Nordeste. Los resultados indican que la experiencia fue positiva para la comprensión de los conceptos propios de la asignatura, dándose además una leve mejoría en el desempeño de los alumnos.
Palabras Claves: Vocaciones STEM Didáctica de laprogramación.Herramientas lúdicas.

\section{INTRODUCCIÓN}

\section{Formación STEM}

Las carreras STEM (Science, Technology, Engineering, Mathematics) están identificadas como las carreras del futuro, y con frecuencia se señala que los países que no desarrollen estas áreas comprometerán su futuro, por lo que, promover la matriculación en ellas es objetivo primordial para aquellas naciones que no deseen estancarse.

Dennis Vilorio [1], economista de la Oficina de Estadísticas Ocupacionales y Proyecciones de Empleo en estados Unidos, rescata lo que dice el director ejecutivo de la Coalición de Educación en STEM, James Brown: "El futuro de la economía está en STEM". Vilorio señala además que los datos de la Oficina de Estadísticas de Trabajo de esa nación (BLS) apoya esta afirmación, sosteniendo que se proyecta que crezca 
a más de 9 millones entre 2012 y 2022 el empleo en ocupaciones relacionadas con STEM. Por otra parte, en ese país, menos del $40 \%$ de los estudiantes que ingresan a la universidad con la intención de especializarse en un campo STEM lo logran [2].

Si bien no existe consenso en cuanto a qué profesiones se consideran parte de los estudios STEM, ya que esto varía de país a país, los expertos están de acuerdo en que los trabajadores entrenados en esta línea utilizan sus conocimientos de la ciencia, tecnología, ingeniería o matemáticas para tratar de entender cómo funciona el mundo y resolver problemas. Esto frecuentemente implica el uso de computadoras $\mathrm{y}$ otras herramientas tecnológicas [3].

En particular, en relación a carreras relacionadas con las Tecnologías de Información y Comunicación (TIC), se observa a nivel mundial una marcada disminución de estudiantes en estas carreras, y escasez de recursos humanos calificados. Numerosos estudios sobre esta problemática intentan determinar las causas y contribuir en la búsqueda de soluciones. Entre ellos destacan:

- La consultora Everis publicó un estudio [3] del cual se desprende que en 2019 habrá un 40\% menos de ingenieros y técnicos informáticos en España.

- Un estudio realizado en la Universidad de California destaca un gran descenso en la elección de Ciencias de la Computación (CS) en la enseñanza superior [4].

- También en California [5], se encuestó a estudiantes del nivel secundario que presentaban aptitudes para las CS, pero que no optaban por ellas. Los resultados indicaron que el $80 \%$ de los alumnos tenían poco co- nocimiento sobre lo que se estudia en CS.

- La National Center for Women E IT (NCWIT) señala que para el 2018 sólo podrán satisfacer un tercio de los trabajos relacionados con tecnologías [6].

- En Argentina, el Reporte Anual sobre el sector de Software y Servicios Informáticos expone los resultados de la encuesta del Observatorio Permanente de la Industria del Software y Servicios Informáticos (OPSSI) perteneciente a la Cámara de la Industria Argentina del Software (CESSI). En él se observa para el 2015 un aumento promedio del empleo del 5,6\%, un crecimiento en las ventas del $29,8 \%$ en pesos, y un aumento del $12,7 \%$ de exportaciones en dólares respecto del 2014. Sin embargo, el presidente de CESSI, Aníbal Carmona, señala que es necesario "encontrar soluciones a la escasez de talentos, que continua siendo uno de los mayores desafíos a resolver para sostener el crecimiento de esta industria" [7].

Ante este problema de carácter global, existen numerosos programas [8] y artículos que proponen posibles soluciones, que buscan aumentar la conciencia entre los estudiantes de la importancia y el atractivo de las carreras de STEM, mejorar la educación en estos temas desde los primeros niveles de educación ("Some STEM for All”), concentrar los esfuerzos sólo en los interesados o en los destacados ("All STEM forSome") [9], fortalecer la formación de los docentes de STEM y promover el incremento de los mismos mediante beneficios adicionales, entre otras [10].

En Argentina también existen políticas para el apoyo y promoción de vocaciones en éstas áreas. Entre estas pueden mencionarse el Plan Estratégico de Formación de 
Ingenieros [11], Proyecto de Mejora de la Formación en Ciencias Exactas y Naturales en la Escuela Secundaria [12], Becas Jóvenes Profesionales TIC, entre otras [13].

Por su parte, la Fundación Sadosky lleva adelante diversos programas, entre estos, Vocaciones TIC y Program.ar. El programa Vocaciones TIC tiene como propósito despertar interés en los jóvenes para estudiar carreras vinculadas con las TIC, en tanto la iniciativa Program. AR tiene como objetivo llevar la enseñanza de las Ciencias de la Computación a los niveles no universitarios [14].

\section{Problemática del ingreso en carre- ras STEM}

Los jóvenes por lo general son renuentes a las carreras que cuentan en su currícula con Algebra, Física, Química, entre otras, y prefieren orientarse hacia carreras sociales, comunicativas, etc., por considerarlas más accesibles a sus capacidades. Las carreras "duras" como pueden ser las ingenierías y otras relacionadas con las ciencias exactas, sufren en muchos países del mundo una baja en sus niveles de inscripción[15].

Por otro lado, en la actualidad las profesiones con mejores campos de trabajo y desarrollo son las relacionadas con las ciencias, la tecnología, ingeniería y las matemáticas. Es paradójico lo que ocurre en el campo de estas carreras. Mientras se progresa hacia una sociedad cada vez más dependiente de estos conocimientos, es decir las empresas necesitan de personas especializadas para poder producir productos y servicios relacionados a la era de la conectividad, en las universidades se presenta el problema de que no se están graduando suficientes individuos con estas calificaciones tan importantes [16].
Por otra parte, se reconocen las dificultades que los alumnos al inicio de las carreras que se traducenen unbajo rendimiento [17].

Es por ello que las universidadesdeberían centrar su atención en esta problemática que conlleve a una reflexión sobre ciertas características que presentan los noveles ingresantes y los nuevos paradigmas que se han implantado en los primeros niveles de las carreras de grado. Las reglas del juego han cambiado, desde cómo educar, buscar un trabajo, promover o financiar emprendimientos [16].

Para lograr el éxito, es imperativo entender y optimizar las nuevas herramientas de que favorezcan la comprensión y asimilación de contenidos académicos en las carreras de las llamadas ciencias duras.

\section{Iniciativas institucionales desde el} 2012

La FaCENA, conciente de la problemática que conlleva el tránsito del estudiante del nivel medio al universitario, propone diversas estrategias para mejorar la experiencia de incorporación de los alumnos. Del 2009 al 2013implementó el Curso de Nivelación y Ambientación para los aspirantes a las 13 carreras que conforman su oferta académica, consistente en tres módulos: Matemática, Leer y escribir para aprender en la universidad, y un curso específico, según la carrera elegida: Algebra, Biología, Física, Química o Informática [18].

Si bien esta modalidad arrojó resultados favorables, el alto número de ingresantes (un promedio de 1500 alumnos), tornaba engorrosa la gestión del dictado de los cursos en forma conjunta para las 13 carreras. Además, cada curso exigía un 
equipo docente contratado para tal fin, con el consiguiente costo presupuestario.

En el año 2013 se diseñó el Programa de Seguimiento a los Ingresantesen articulación con los espacios de Tutorías y el Gabinete Psicopedagógico, con el propósito de acompañar al ingresante, mejorando sus posibilidades de incorporarse y permanecer en la carrera elegida.Para ello se designaron espacios y horarios permantentes de apoyo tutoriales y se coordinaron actividades desde las distintas cátedras con los tutores de acuerdo a las dificultades detectadas, organizando talleres, charlas y seguimiento personalizado.

En el año 2014, el dictado de las asignaturas se inició en forma anticipada para los alumnos ingresantes, con el objetivo de disponer de un período de aprestamiento de 3 semanas, previo al dictado de los contenidos propios de la materia específica del primer cuatrimestre del primer año de cada una de las carreras. Este periodo de aprestamiento tiene como objetivos:

- Acompañar a los estudiantes a la adaptación progresiva a la dinámica universitaria

- Generar espacios de enseñanza con un aumento gradual de las exigencias de la apropiación conceptual

- Favorecer la reactivación de conocimientos previos, en un trayecto de complejidad creciente y de progresivo acercamiento a los desafíos cognitivos exigidos en la educación superior

- Promover y orientar la revisión de elecciones vocacionales.
Cada asignatura planificó el periodo de aprestamiento considerando las dificultades usuales de los alumnos en la misma. Por tanto, las actividades se enfocaron en repasar y consolidar contenidos fundamentales dados en el nivel medio, como así también, se buscó articular con los contenidos universitarios en un sentido amplio, es decir no sólo lo referido a lo conceptual sino también a lo procedimental y actitudinal, consolidando competencias transversales necesarias para una adaptación exitosa a la vida universitaria. Como ventaja de esta modalidad los estudiantes toman contacto tempranamente con los docentes de la asignatura, a diferencia de la modalidad del curso de ingreso que requería un equipo docente contratado ad hoc, con escasa o nula articulación con las asignaturas de la carrera.

\section{Experiencia innovadora}

En el año 2016, la asignatura Algoritmos y Estructura de Datos I, del primer cuatrimestre del primer año de la carrera Licenciatura en Sistemas de Información, incorporó en su periodo de aprestamiento actividades lúdicas de programación utilizando las herramientas Lightbot y Scratch, con el objetivo de motivar a los alumnos y lograr una mejor comprensión de los conceptos básicos de programación que forman parte de los contenidos propios de la asignatura.

Las actividades del aprestamiento se tomaron del curso Introducción a la Programación y su Didáctica, que la Fundación Sadosky propone para la formación de docentes a través de la iniciativa Program.Ar [19].

El periodo de aprestamiento se desarrolló desde el 22 de febrero al 11 de marzo de 2.016, mediante 2 clases semanales de 2 hs. En la propuesta formativa se insistió 
en los conceptos de "abstracción", esto es, pensar una estrategia antes de intentar la resolución de los ejercicios, la "modularización o descomposición del problema en partes", traducida en la creación de bloques en la solución diseñada y en la "legibilidad" de la solución, mediante la definición de nombres representativos a los bloques.

Luego del periodo de aprestamiento la asignatura desarrolló sus contenidos específicos: introducción a la programación estructurada utilizando el lenguaje de programación $\mathrm{C}$ para el desarrollo de los prácticos. Durante el dictado, al introducir los distintos conceptos (estructuras de control,funciones, parámetros, recorrido en arreglos), se buscó relacionar con las actividades de Scracht en las que se utilizaba técnicas similares.

Al finalizar el dictado, se realizó una encuesta a los alumnos, con el propósito de determinar si el conocimiento adquirido con las actividades del periodo de aprestamiento, le resultó útil para una mejor comprensión de los conceptos y de las habilidades necesarias para el logro de los objetivos de aprendizaje de la asignatura. Los resultados fueron publicados en [20].En relación a la estrategia de innovación, se destacan los siguientes resultados:

Se preguntó a los alumnos en qué grado las actividades realizadas durante el aprestamiento contribuyeron a que incorporaranlos aspectos claves que se tienen en cuenta para resolver problemas, tales como:

- Abstracción: Pensar una estrategia antes de revolver los ejercicios.

- Modularización: Descomponerel problema en partes

- Legibilidad: Dar nombres repre- sentativos a los módulos

Los alumnos señalaron un aporte significativo en todos los aspectos: $68 \%$ en la abstracción, $75 \%$ en la modularización y $71 \%$ en la legibilidad, como se ve en la figura 1.

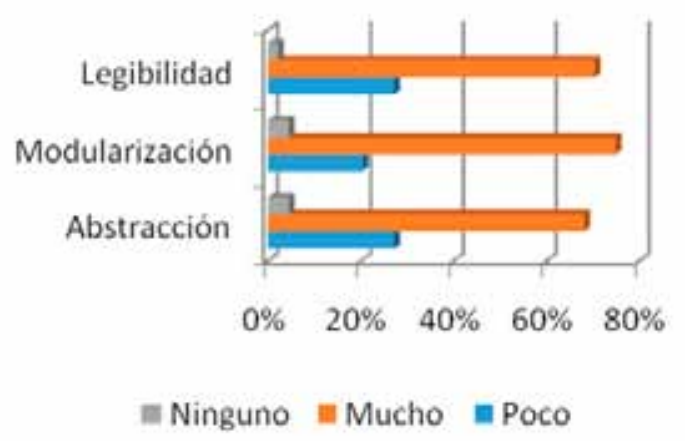

Figura 1. Grado de aporte a los aspectos claves del método para resolver problemas.

También se preguntó a los alumnos en qué grado las actividades del aprestamiento lo ayudaron a comprender los conceptos fundamentales de la programación, tales como:

- Secuencia (Órdenes)

- Alternativa (Selección)

- Iteración (Repetición)

- Modularización (Uso de funciones)

- Parámetros (Generalización)

Al respecto, los alumnos valoraron el aporte de las actividades desarrolladas durante el aprestamiento para la comprensión de los conceptos mencionados. Se destaca que el mayor aporte fue en Iteración (91\%). En tanto, la menor contribución fue en Parametrización, en el cuál el $50 \%$ de los alumnos manifestó que le re- 
sultó poco útil, como se puede apreciar en la figura 2. Este es un concepto complejo para los alumnos, y el breve periodo asignado no alcanzó para consolidar el concepto.

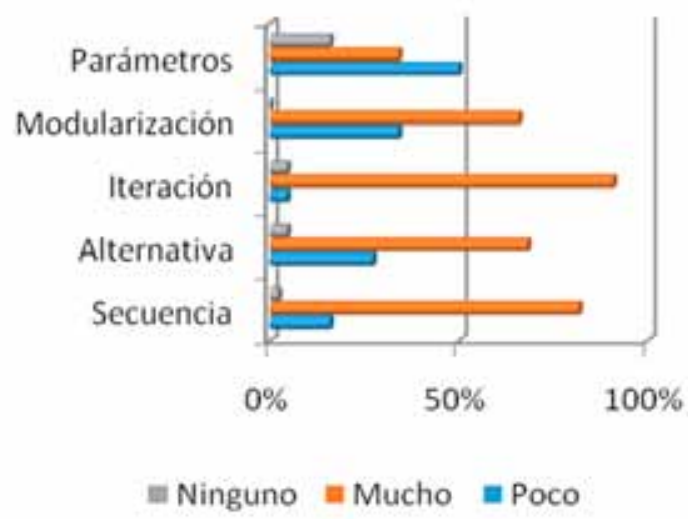

Figura2. Grado de aporte a lacomprensión de los conceptos fundamentales en programación

Tabla 1. Rendimiento académico Algoritmos y Estructuras de Datos I 2015-2016

\begin{tabular}{|l|r|r|r|r|}
\hline Condición & 2015 & $\% /$ Tot & 2016 & $\% /$ Tot \\
\hline Regularizaron & 68 & $31 \%$ & 70 & $31 \%$ \\
\hline Promocionaron & 52 & $24 \%$ & 59 & $26 \%$ \\
\hline Libres por parciales & 54 & $25 \%$ & 26 & $12 \%$ \\
\hline Libres por asistencia & 43 & $20 \%$ & 68 & $30 \%$ \\
\hline Total de cursantes & 217 & & 223 & \\
\hline
\end{tabular}

Finalmente, analizado el rendimiento académico de los últimos 2 añosse puede observar una leve mejoría en el porcentaje de alumnos que promocionó la asignatura, como se muestra en la tabla 1.

\section{CONCLUSIONES}

Las facultades cuya oferta académica están vinculadas con las disciplinas STEM, tienen que visibilizar esta problemática de las carreras de las "ciencias duras"y continuar en la búsqueda permanente de nuevos métodos parafavorecer el ingreso y permanencia de los alumnos en estas carreras cuya formación es altamente demandada por su contribución en el desarrollo de los países.

En la carrera Licenciatura en Sistemas de Información se implementó una modalidad de aprestamiento innovadora para mejorar el inicio de la carrera de los alumnos del primer año. Los resultados indican que la experiencia fue positiva para la comprensión de los conceptos propios de la asignatura, dándose además una leve mejoría en el desempeño de los alumnos.

A futuro, se pretende continuar con esta propuesta, incorporando mejoras en función de la retroalimentación de la experiencia realizada y evaluando el impacto en el proceso de enseñanza y aprendizaje. 


\section{Bibliografía}

[1] Vilorio, D. STEM 101: Intro to tomorrow's jobs. Occupational Outlook Quarterly. Spring 2014. Disponibleen:

http://www.stemedcoalition.org/wp-content/ uploads/2010/05/BLS-STEM-Jobs-reportspring-2014.pdf

[2]President's Council of Advisors on Science and Technology. (2012). "Report to the president engage to excel: producing one million additional college graduates with degrees in science, technology, engineering, and mathematics". Disponible en: http://www. whitehouse.gov/sites/default/files/microsites/ostp/ pcast-engage-to-excel-final_2-25-12.pdf

[3] Consultora EVERIS. (2012) "La falta de ingenieros TIC: situación actual y perspectiva”. Barcelona. Disponible en: http://www.everis.com/spain/WCLibraryRepository/La\%20falta\%20de\%20ingenieros. pdf

[4]Vegso, J., (2005). "Interest in CS as a Major Drops Among Incoming Freshmen”. Computing Research News, Vol. 17/No.3,

[5] Carter, L. (2006). "Why Students with an Apparent Aptitude for Computer Science Don't Choose to Major in Computer Science”. SIGCSE’06.

[6] National Center for Women \& IT.(2011). "A report on the status of women in information technology". Disponibleen:

https://www.ncwit.org/sites/default/files/resources/ ncwitscorecard_05152015_lowres.pdf

[7] CESSI. La industria argentina del software creció en 2015 y espera crear 7000 nuevos empleos en 2016. Disponible en:

http://www.cessi.org.ar/ver-noticias-la-industria-argentina-del-software-crecio-en-2015-y-espera-crear7-000-nuevos-empleos-en-2016-2009

[8] SHPE Foundation - Advancing Hispanic Excellence in Technology, Engineering, Mathematics and Science. http://www.shpe.org/

[9] Atkinson, R.D.; Mayo, M."Refueling the U.S. innovation economy: Fresh Approaches to Science, Technology, Engineering and Mathematics (STEM) Education". Disponible en:http://www.itif.org/ files/2010-refueling-innovation-economy.pdf

[10] Committee Members. "Rising above the gathering storm, revisited”. Disponible en: http://www.sandia.
gov/NINE/documents/RisingAbove.pdf

[11] SPU. Plan Estratégico de Formación de Ingenieros. Disponible en:

http://portales.educacion.gov.ar/spu/calidad-universitaria/plan-estrategico-de-formacion-de-ingenieros-2012-2016/

[12] SPU. La Universidad y la Escuela secundaria. Mejora de la formación en Ciencias Exactas y Naturales. Disponible en: http://portales.educacion.gov.ar/spu/ la-universidad-y-la-escuela-secundaria/

[13] Programa para promover la Innovación Productiva. Componente Formación de Recursos Humanos. Disponible en: http://www.agencia.mincyt.gob.ar/ upload/Bases_Becas.pdf

[14]Program.Ar.FundaciónSadosky delMinisterio de Ciencia, Tecnología e Innovación Productiva. Disponible en: http://program.ar/quienes-somos/

[15] Daniel Giulianelli, Rocío Rodríguez, Pablo Vera, Artemisa Trigueros, Graciela Cruzado, Edgardo Moreno, Isabel Marko (2014). Incorporación de Recursos Audiovisuales como Apoyo al

Aprendizaje en carreras de Ingeniería. XX Congreso Argentino de Ciencias de la Computación. Buenos Aires.

[16] Ariel Coro. Carreras STEM: Sin raíces no hay tallo. https://tutecnologia.com/carreras-stem-sin-raicesno-hay-tallo/.

[17]Porcel, Eduardo Adolfo, Dapozo, Gladys Noemí, \& López, María Victoria. (2010). Predicción del rendimiento académico de alumnos de primer año de la FACENA (UNNE) en función de su caracterización socioeducativa. Revista electrónica de investigación educativa, 12(2), 1-21.

[18] Dapozo, G.N.; Pelozo, S.S (2009). Implementación del módulo Informática para los ingresantes a la carrera Licenciatura en Sistemas de Información de la UNNE. Revista Iberoamericana de Tecnología en Educación y Educación en Tecnología. Vol.4.

[19] Pablo Matías Factorovich, Federico A. SawadyO'Connor (2015). Actividades para aprender a Program.AR Volumen 1. E-Book. (Program.AR) ISBN 978-987-27416-1-7.

[20] Gladys Dapozo; Cristina Greiner, Raquel Petris (2016). "Herramientas lúdicas como apoyo a la enseñanza de la programación”. Anales del Congreso Argentino de Computación (CACIC 2016). San Luis. 